\title{
On Franel-Kluyver integrals of order three
}

by

\author{
J. C. Wilson (York)
}

1. Introduction. In 1924 Franel [2] proved the formula

$$
\frac{1}{a b} \int_{0}^{a b}\left(\left\{\frac{x}{a}\right\}-\frac{1}{2}\right)\left(\left\{\frac{x}{b}\right\}-\frac{1}{2}\right) d x=\frac{(a, b)}{12[a, b]}
$$

(where $\{x\}$ denotes the fractional part of $x$ ), which he used to establish a connection between the Riemann hypothesis and the distribution of Farey sequences.

In Greaves, Hall, Huxley and Wilson [3] we defined the Franel integral of order $n$ by

$$
J\left(a_{1}, \ldots, a_{n}\right)=\frac{1}{a_{1} \ldots a_{n}} \int_{0}^{a_{1} \ldots a_{n}} \varrho\left(\frac{x}{a_{1}}\right) \ldots \varrho\left(\frac{x}{a_{n}}\right) d x,
$$

where $a_{1}, \ldots, a_{n}$ are positive integers and $\varrho(x)=[x]-x+1 / 2$. In particular, for $n=4$, we evaluated certain cases in terms of elementary functions of the h.c.f.'s and l.c.m.'s of $a_{1}, \ldots, a_{n}$ : others involved generalized Dedekind sums and related cotangent sums.

In fact, twenty years before Franel proved equation (1), Kluyver [6] had implicitly proved the more general result

(2) $\quad \frac{1}{a b} \int_{0}^{a b} \bar{B}_{m}\left(\frac{x}{a}\right) \bar{B}_{n}\left(\frac{x}{b}\right) d x=(-1)^{n-1} \frac{m ! n !}{(m+n) !} B_{m+n} \frac{(a, b)^{m+n}}{a^{m} b^{n}}$

for all positive integers $m, n, a, b$. Here $\bar{B}_{r}(x)$ is the periodic extension into $\mathbb{R}$ of the Bernoulli polynomial $B_{r}(x)$ on $[0,1)$ given by the relation

$$
\frac{z e^{x z}}{e^{z}-1}=\sum_{r=0}^{\infty} B_{r}(x) \frac{z^{r}}{r !} \quad(|z|<2 \pi) .
$$

In this paper we generalize the Franel integral of order 3 in two ways. Firstly, following Kluyver, we replace the function $\varrho$ by higher order Bernoulli func- 
tions and define the Franel-Kluyver integral of order 3 by

$$
J_{l, m, n}\left(a_{1}, a_{2}, a_{3}\right)=\frac{1}{a_{1} a_{2} a_{3}} \int_{0}^{a_{1} a_{2} a_{3}} \bar{B}_{l}\left(\frac{x}{a_{1}}\right) \bar{B}_{m}\left(\frac{x}{a_{2}}\right) \bar{B}_{n}\left(\frac{x}{a_{3}}\right) d x
$$

(where $l+m+n \equiv 0(\bmod 2)$ : if $l+m+n$ is odd then the integrand is an odd, periodic function and the integral is zero). We show that this integral can be evaluated as a linear combination of the generalized Dedekind sums

$$
S_{m, n}(h, k)=\sum_{r=0}^{k-1} \bar{B}_{m}\left(\frac{r}{k}\right) \bar{B}_{n}\left(\frac{r h}{k}\right) .
$$

Secondly, we define

$$
J\left(a_{1}, a_{2}, a_{3} ; \theta\right)=\frac{1}{a_{1} a_{2} a_{3}} \int_{0}^{a_{1} a_{2} a_{3}} \bar{B}_{1}\left(\frac{x}{a_{1}}\right) \bar{B}_{1}\left(\frac{x}{a_{2}}\right) \bar{B}_{1}\left(\frac{x}{a_{3}}+\theta\right) d x .
$$

The evaluation of this integral involves the further generalized DedekindRademacher sum

$$
S_{m, n}(h, k ; x)=\sum_{r=0}^{k-1} \bar{B}_{m}\left(\frac{r}{k}\right) \bar{B}_{n}\left(\frac{r h}{k}+x\right) .
$$

(Carlitz [1] has defined $\phi_{m, n}(h, k ; x, y)$ where $S_{m, n}(h, k ; x)=\phi_{n, m}(h, k ; x, 0)$ and proved reciprocity formulae for these sums.)

In Section 2 we show how both (4) and (6) can be reduced to integrals involving the functions $\bar{B}_{r}\left(a_{i} x\right)$, in which we need only consider pairwise coprime variables. We work out the Fourier series for $\bar{B}_{l}(a x) \bar{B}_{m}(b x)$ in Section 3 which we then use to evaluate integrals equivalent to (4) and (6) in Theorems 1 and 2 respectively.

We shall make use of the following alternative expression for the generalized Dedekind sum (5):

$$
S_{m, n}(h, k)=\frac{i^{n-m}}{(2 \pi)^{m+n}} \frac{m n}{k^{m+n-1}} \sum_{r=0}^{k-1} C^{(m)}\left(\frac{r h}{k}\right) C^{(n)}\left(\frac{r}{k}\right),
$$

where we have defined

$$
C^{(m)}(z)=\frac{d^{m}}{d z^{m}} \log (\sin \pi z)=-(m-1) ! \sum_{t=-\infty}^{\infty} \frac{1}{(t-z)^{m}}
$$

for $z \notin \mathbb{Z}$, and

$$
C^{(m)}(0)= \begin{cases}-2 \zeta(m)(m-1) ! & \text { if } m \text { is even, } \\ 0 & \text { if } m \text { is odd. }\end{cases}
$$


Eisenstein proved that

$$
\bar{B}_{1}\left(\frac{r}{k}\right)=\frac{i}{2 \pi k} \sum_{a=0}^{k-1} C^{(1)}\left(\frac{a}{k}\right) e\left(\frac{r a}{k}\right)
$$

$(e(x):=\exp (2 \pi i x))$, so that the ordinary Dedekind sum may be expressed in terms of cotangents. Analogously, using the generalization

$$
\bar{B}_{m}\left(\frac{r}{k}\right)=\frac{m}{k^{m}}\left(\frac{i}{2 \pi}\right)^{m} \sum_{a=0}^{k-1} C^{(m)}\left(\frac{a}{k}\right) e\left(\frac{r a}{k}\right),
$$

we see that

$$
\begin{aligned}
& S_{m, n}(h, k) \\
& =\frac{m n}{k^{m+n}}\left(\frac{i}{2 \pi}\right)^{m+n} \sum_{r=0}^{k-1} \sum_{a=0}^{k-1} \sum_{b=0}^{k-1} C^{(m)}\left(-\frac{a}{k}\right) C^{(n)}\left(\frac{b}{k}\right) e\left(\frac{r h b-r a}{k}\right)
\end{aligned}
$$

which, since

$$
\sum_{r=0}^{k-1} e\left(\frac{r h b-r a}{k}\right)= \begin{cases}k & \text { if } a \equiv h b(\bmod k), \\ 0 & \text { else }\end{cases}
$$

gives (8).

2. Reduction steps and related integrals. We let

$$
J_{l, m, n}\left(a_{1}, a_{2}, a_{3} ; \theta\right)=\frac{1}{a_{1} a_{2} a_{3}} \int_{0}^{a_{1} a_{2} a_{3}} \bar{B}_{l}\left(\frac{x}{a_{1}}\right) \bar{B}_{m}\left(\frac{x}{a_{2}}\right) \bar{B}_{n}\left(\frac{x}{a_{3}}+\theta\right) d x
$$

and define the related integral $I_{l, m, n}\left(a_{1}, a_{2}, a_{3} ; \theta\right)$ by

$$
I_{l, m, n}\left(a_{1}, a_{2}, a_{3} ; \theta\right)=\int_{0}^{1} \bar{B}_{l}\left(a_{1} x\right) \bar{B}_{m}\left(a_{2} x\right) \bar{B}_{n}\left(a_{3} x+\theta\right) d x .
$$

Since the integrand in (12) has period $\left[a_{1}, a_{2}, a_{3}\right]$ we may write (12) as

$$
\begin{array}{r}
J_{l, m, n}\left(a_{1}, a_{2}, a_{3} ; \theta\right) \\
\quad=\frac{1}{\left[a_{1}, a_{2}, a_{3}\right]} \int_{0}^{\left[a_{1}, a_{2}, a_{3}\right]} \bar{B}_{l}\left(\frac{x}{a_{1}}\right) \bar{B}_{m}\left(\frac{x}{a_{2}}\right) \bar{B}_{n}\left(\frac{x}{a_{3}}+\theta\right) d x .
\end{array}
$$

Then, substituting $x=\left[a_{1}, a_{2}, a_{3}\right] y$, we find that

$$
J_{l, m, n}\left(a_{1}, a_{2}, a_{3} ; \theta\right)=I_{l, m, n}\left(A_{1}, A_{2}, A_{3} ; \theta\right)
$$

where

$$
A_{i}=\frac{\left[a_{1}, a_{2}, a_{3}\right]}{a_{i}} \quad(1 \leq i \leq 3)
$$


There is an analogous transformation in the opposite direction.

We now show that the integral in (12) can be reduced to an $I$ integral in which the variables are pairwise coprime. Firstly we may assume the $a_{i}$ in (12) have no common divisor, since putting $x=k y$ gives

$$
\begin{aligned}
& J_{l, m, n}\left(k a_{1}, k a_{2}, k a_{3} ; \theta\right) \\
& =\frac{1}{k^{3} a_{1} a_{2} a_{3}} \int_{0}^{k^{2} a_{1} a_{2} a_{3}} \bar{B}_{l}\left(\frac{y}{a_{1}}\right) \bar{B}_{m}\left(\frac{y}{a_{2}}\right) \bar{B}_{n}\left(\frac{y}{a_{3}}+\theta\right) k d y \\
& =J_{l, m, n}\left(a_{1}, a_{2}, a_{3} ; \theta\right)
\end{aligned}
$$

by periodicity.

We can also write $J_{l, m, n}\left(a_{1}, a_{2}, a_{3} ; \theta\right)$ as

$$
\begin{aligned}
& J_{l, m, n}\left(a_{1}, a_{2}, a_{3} ; \theta\right) \\
& =\frac{1}{a_{1} a_{2} a_{3}} \sum_{s=0}^{a_{1} a_{2} a_{3}-1} \int_{s}^{s+1} \bar{B}_{l}\left(\frac{x}{a_{1}}\right) \bar{B}_{m}\left(\frac{x}{a_{2}}\right) \bar{B}_{n}\left(\frac{x}{a_{3}}+\theta\right) d x \\
& =\frac{1}{a_{1} a_{2} a_{3}} \int_{0}^{1} \sum_{s=0}^{a_{1} a_{2} a_{3}-1} \bar{B}_{l}\left(\frac{s+y}{a_{1}}\right) \bar{B}_{m}\left(\frac{s+y}{a_{2}}\right) \bar{B}_{n}\left(\frac{s+y}{a_{3}}+\theta\right) d y .
\end{aligned}
$$

If we now let $K=\left[a_{1}, a_{2}\right]$ and $k=\left(a_{3}, K\right)$, where $a_{3}=h k$, then $h K=$ $\left[a_{1}, a_{2}, a_{3}\right]$ and, by periodicity,

$$
\begin{array}{r}
J_{l, m, n}\left(a_{1}, a_{2}, a_{3} ; \theta\right) \\
=\frac{1}{h K} \int_{0}^{1} \sum_{s=0}^{h K-1} \bar{B}_{l}\left(\frac{s+y}{a_{1}}\right) \bar{B}_{m}\left(\frac{s+y}{a_{2}}\right) \bar{B}_{n}\left(\frac{s+y}{a_{3}}+\theta\right) d y .
\end{array}
$$

We write $s=t K+u$, for $0 \leq u \leq K-1$ and $0 \leq t \leq h-1$; then

$$
\begin{aligned}
& J_{l, m, n}\left(a_{1}, a_{2}, a_{3} ; \theta\right) \\
& \quad=\frac{1}{h K} \int_{0}^{1} \sum_{t=0}^{h-1} \sum_{u=0}^{K-1} \bar{B}_{l}\left(\frac{u+y}{a_{1}}\right) \bar{B}_{m}\left(\frac{u+y}{a_{2}}\right) \bar{B}_{n}\left(\frac{t K+u+y}{h k}+\theta\right) d y .
\end{aligned}
$$

Now $(h, K / k)=1$, so that if $t$ runs through all residue classes modulo $h$, then so does $t K / k$ and

$$
\begin{aligned}
\sum_{t=0}^{h-1} \bar{B}_{n}\left(\frac{t K+u+y}{h k}+\theta\right) & =\sum_{t=0}^{h-1} \bar{B}_{n}\left(\frac{t}{h}+\frac{u+y+h k \theta}{h k}\right) \\
& =\frac{1}{h^{n-1}} \bar{B}_{n}\left(\frac{u+y}{k}+h \theta\right) .
\end{aligned}
$$

Thus 


$$
\begin{aligned}
& J_{l, m, n}\left(a_{1}, a_{2}, a_{3} ; \theta\right) \\
& \quad=\frac{1}{h^{n} K} \int_{0}^{1} \sum_{u=0}^{K-1} \bar{B}_{l}\left(\frac{u+y}{a_{1}}\right) \bar{B}_{m}\left(\frac{u+y}{a_{2}}\right) \bar{B}_{n}\left(\frac{u+y}{k}+h \theta\right) d y \\
& =\frac{1}{h^{n}} J_{l, m, n}\left(a_{1}, a_{2}, k ; h \theta\right) .
\end{aligned}
$$

It is convenient at this stage to introduce the idea of total decomposition sets, which we now define.

With any positive integers $a_{1}, \ldots, a_{n}$ we associate $2^{n}-1$ further positive integers $d(S)$, where $S$ runs through the non-empty subsets of $\{1, \ldots, n\}$, having the properties:

(i) For any non-empty $T \subseteq\{1, \ldots, n\}$ we have

$$
\text { h.c.f. }\left(a_{i}: i \in T\right)=\prod\{d(S): T \subseteq S\} \text {. }
$$

(ii) For any non-empty $T$ we have

$$
\text { l.c.m. }\left[a_{i}: i \in T\right]=\prod\{d(S): S \cap T \neq \emptyset\} .
$$

We refer to $\{d(S)\}$ as the total decomposition set of $\left\{a_{1}, \ldots, a_{n}\right\}$. Its existence and uniqueness were established in [4].

We shall also make use of the following lemma, which was proved by Hall in [5].

Lemma 1. Let $2^{n}-1$ positive integers $e(S)$ be given, where $S$ runs through the non-empty subsets of $\{1, \ldots, n\}$. Then $\{e(S)\}$ is a total decomposition set if, and only if, for every pair of subsets $R, S$ neither of which contains the other, we have $(e(R), e(S))=1$.

Using this notation we can write

$$
a_{1}=d_{1} d_{12} d_{13} d_{123}, \quad a_{2}=d_{2} d_{12} d_{23} d_{123}, \quad a_{3}=d_{3} d_{13} d_{23} d_{123},
$$

and it follows from (16) and (17) that

$$
J_{l, m, n}\left(a_{1}, a_{2}, a_{3} ; \theta\right)=\frac{1}{d_{1}^{l} d_{2}^{m} d_{3}^{n}} J_{l, m, n}\left(d_{12} d_{13}, d_{12} d_{23}, d_{13} d_{23} ; d_{3} \theta\right) .
$$

Now, by Lemma 1,

$$
\left[d_{12} d_{13}, d_{12} d_{23}, d_{13} d_{23}\right]=d_{12} d_{13} d_{23}
$$

so that, from (15),

$$
J_{l, m, n}\left(a_{1}, a_{2}, a_{3} ; \theta\right)=\frac{1}{d_{1}^{l} d_{2}^{m} d_{3}^{n}} I_{l, m, n}\left(d_{23}, d_{13}, d_{12} ; d_{3} \theta\right)
$$

and we notice that the variables $d_{23}, d_{13}$ and $d_{12}$ are pairwise relatively prime. 
3. The Fourier series for $\bar{B}_{l}(a x) \bar{B}_{m}(b x)$. Let $a$ and $b$ be coprime positive integers. The function $\bar{B}_{l}(a x) \bar{B}_{m}(b x)$ has period 1 and so has an expansion in complex Fourier series

$$
\bar{B}_{l}(a x) \bar{B}_{m}(b x) \sim \sum_{k=-\infty}^{\infty} c_{k}(a, b) e(k x)
$$

where

$$
c_{k}(a, b)=\int_{0}^{1} \bar{B}_{l}(a x) \bar{B}_{m}(b x) e(-k x) d x .
$$

We apply Parseval's theorem to the functions $\bar{B}_{l}(a x)$ and $\bar{B}_{m}(b x) e(-k x)$. (See Whittaker and Watson [8], §9.5.) Since

$$
\bar{B}_{r}(x) \sim-r ! \sum_{n=-\infty}^{\infty} \frac{e(n x)}{(2 \pi i n)^{r}}
$$

(where the dash denotes throughout that undefined terms are excluded from the sum) this gives

$$
\begin{aligned}
c_{k}(a, b) & =\frac{l ! m !}{(2 \pi i)^{l+m}} \sum_{g a+h b-k=0} \sum_{g^{l} h^{m}} \\
& =\frac{l ! m !}{(2 \pi i)^{l+m}} \sum_{d=-\infty}^{\infty} \frac{1}{(k \bar{a}+d b)^{l}(k \bar{b}-d a)^{m}},
\end{aligned}
$$

where $\bar{a} a+\bar{b} b=1$. Now

$$
\sum_{d=-\infty}^{\infty} \frac{1}{d^{r}}= \begin{cases}2 \zeta(r)=-\frac{(2 \pi i)^{r} B_{r}}{r !} & \text { if } r \text { is even }, \\ 0 & \text { if } r \text { is odd } .\end{cases}
$$

Therefore, since $B_{r}=0$ for odd $r>1$, we may write

$$
\sum_{d=-\infty}^{\infty} \frac{1}{d^{r}}=-\frac{(2 \pi i)^{r} B_{r}}{r !}
$$

for any $r>1$. Hence

$$
c_{0}(a, b)=\frac{(-1)^{m-1} l ! m ! B_{l+m}}{a^{m} b^{l}(l+m) !} .
$$

To find $c_{k}(a, b)$ for $k \neq 0$, we consider the integral

$$
\frac{1}{2 \pi i} \int_{Q_{M}} f(z) d z
$$


where

$$
f(z)=\frac{\pi \cot (\pi z)}{(k \bar{a}+z b)^{l}(k \bar{b}-z a)^{m}}
$$

and $Q_{M}$ is the square with corners $(M+1 / 2)( \pm 1 \pm i)$. We note that $k \bar{b} / a \neq$ $-k \bar{a} / b$ for $k \neq 0$ and let $R_{1}$ and $R_{2}$ be the residues at $z=-k \bar{a} / b$ and $z=k \bar{b} / a$ respectively. Then, since the integral round $Q_{M}$ tends to zero as $M \rightarrow \infty$, we have

$$
0=\lim _{M \rightarrow \infty} \sum_{l=-M}^{M} \frac{1}{(k \bar{a}+d b)^{l}(k \bar{b}-d a)^{m}}+R_{1}+R_{2}
$$

and so, for $k \neq 0$,

$$
c_{k}(a, b)=-\frac{l ! m !}{(2 \pi i)^{l+m}}\left(R_{1}+R_{2}\right) .
$$

We consider the different cases separately.

Case 1: $a \nmid k, b \nmid k$. Since $f(z)$ has a pole of order $l$ at $z=-k \bar{a} / b$, we have

$$
\begin{aligned}
R_{1}= & \frac{1}{(l-1) !} \frac{d^{l-1}}{d z^{l-1}}\left\{\frac{\pi \cot (\pi z)}{b^{l}(k \bar{b}-z a)^{m}}\right\}_{z \rightarrow-k \bar{a} / b} \\
= & \frac{1}{a^{m} b^{l}(l-1) !} \\
& \times\left\{\sum_{s=0}^{l-1}\left(\begin{array}{c}
l-1 \\
s
\end{array}\right) \frac{d^{l-1-s}}{d z^{l-1-s}} C^{(1)}(z) \frac{d^{s}}{d z^{s}}\left(\frac{k \bar{b}}{a}-z\right)^{-m}\right\}_{z \rightarrow-k \bar{a} / b} \\
= & \frac{1}{a^{m} b^{l}(l-1) !} \\
& \times\left\{\sum_{s=0}^{l-1}\left(\begin{array}{c}
l-1 \\
s
\end{array}\right) C^{(l-s)}(z) \frac{(m+s-1) !}{(m-1) !}\left(\frac{k \bar{b}}{a}-z\right)^{-(m+s)}\right\}_{z \rightarrow-k \bar{a} / b} \\
= & \frac{(-1)^{l} b^{m-l}}{k^{m}(l-1) !(m-1) !} \\
& \times \sum_{s=0}^{l-1}\left(\begin{array}{c}
l-1 \\
s
\end{array}\right)(-1)^{s}(m+s-1) !\left(\frac{a b}{k}\right)^{s} C^{(l-s)}\left(\frac{k \bar{a}}{b}\right) .
\end{aligned}
$$

Similarly,

$$
R_{2}=\frac{(-1)^{m} a^{l-m}}{k^{l}(l-1) !(m-1) !} \sum_{s=0}^{m-1}\left(\begin{array}{c}
m-1 \\
s
\end{array}\right)(-1)^{s}(l+s-1) !\left(\frac{a b}{k}\right)^{s} C^{(m-s)}\left(\frac{k \bar{b}}{a}\right)
$$


and so, when $a \nmid k, b \nmid k$, we have

$$
\begin{aligned}
& c_{k}(a, b)=\frac{l m}{(2 \pi i)^{l+m}} \\
& \quad \times\left\{\frac{b^{m-l}}{k^{m}} \sum_{s=0}^{l-1}\left(\begin{array}{c}
l-1 \\
s
\end{array}\right)(-1)^{l+s-1}(m+s-1) !\left(\frac{a b}{k}\right)^{s} C^{(l-s)}\left(\frac{k \bar{a}}{b}\right)\right. \\
& \left.\quad+\frac{a^{l-m}}{k^{l}} \sum_{s=0}^{m-1}\left(\begin{array}{c}
m-1 \\
s
\end{array}\right)(-1)^{m+s-1}(l+s-1) !\left(\frac{a b}{k}\right)^{s} C^{(m-s)}\left(\frac{k \bar{b}}{a}\right)\right\} .
\end{aligned}
$$

Case 2: $a \mid k$ but $b \nmid k$. At $z=-k \bar{a} / b, f(z)$ has a pole of order $l$, with residue

$$
\begin{aligned}
R_{1}=\frac{(-1)^{l} b^{m-l}}{k^{m}(l-1) !}(m-1) ! \\
\quad \times \sum_{s=0}^{l-1}\left(\begin{array}{c}
l-1 \\
s
\end{array}\right)(-1)^{s}(m+s-1) !\left(\frac{a b}{k}\right)^{s} C^{(l-s)}\left(\frac{k \bar{a}}{b}\right) .
\end{aligned}
$$

Also $f(z)$ has a pole of order $m+1$ at $z=k \bar{b} / a$. We put $z=w+k \bar{b} / a$; then the Laurent expansion becomes

$$
\begin{aligned}
\frac{c_{-(m+1)}}{w^{m+1}}+\ldots+\frac{c_{-1}}{w}+\ldots & =\frac{\pi \cot (\pi w+\pi k \bar{b} / a)}{(k \bar{a}+w b+k \bar{b} b / a)^{l}(-w a)^{m}} \\
& =\frac{\pi \cot (\pi w)}{(-w)^{m} k^{l} a^{m-l}(1+a b w / k)^{l}} .
\end{aligned}
$$

We use the expansion

$$
\pi \cot (\pi w)=\sum_{h=0}^{\infty} \frac{(2 \pi i)^{2 h} B_{2 h} w^{2 h-1}}{(2 h) !}
$$

so that we require the coefficient of $w^{m-1}$ in

$$
\frac{(-1)^{m}}{k^{l} a^{m-l}} \sum_{h=0}^{\infty} \frac{(2 \pi i)^{2 h} B_{2 h} w^{2 h-1}}{(2 h) !} \sum_{g=0}^{\infty}\left(\begin{array}{c}
g+n-1 \\
g
\end{array}\right)\left(-\frac{a b w}{k}\right)^{g} .
$$

This is

$$
\frac{1}{k^{l} a^{m-l}} \sum_{r=0}^{m / 2} \frac{(2 \pi i)^{m-2 r} B_{m-2 r}}{(m-2 r) !}\left(\begin{array}{c}
l+2 r-1 \\
2 r
\end{array}\right)\left(\frac{a b}{k}\right)^{2 r}
$$

when $m$ is even, and

$$
\frac{1}{k^{l} a^{m-l}} \sum_{r=0}^{(m-1) / 2} \frac{(2 \pi i)^{m-1-2 r} B_{m-1-2 r}}{(m-1-2 r) !}\left(\begin{array}{l}
l+2 r \\
2 r+1
\end{array}\right)\left(\frac{a b}{k}\right)^{2 r+1}
$$


when $m$ is odd. Again, since $B_{r}=0$ for odd $r>1$, we can write

$$
R_{2}=\frac{1}{k^{l} a^{m-l}} \sum_{s=0}^{m-2} \frac{(2 \pi i)^{m-s} B_{m-s}}{(m-s) !}\left(\begin{array}{c}
l+s-1 \\
s
\end{array}\right)\left(\frac{a b}{k}\right)^{s}+\frac{a^{l} b^{m}}{k^{l+m}}\left(\begin{array}{c}
l+m-1 \\
m
\end{array}\right)
$$

for any $m$. Hence, when $a \mid k$ but $b \nmid k$, we have

$$
\begin{aligned}
& c_{k}(a, b) \\
= & \frac{a^{l-m} l m}{k^{l}(2 \pi i)^{l+m}} \sum_{s=0}^{m-1}\left(\begin{array}{c}
m-1 \\
s
\end{array}\right)(-1)^{m+s-1}(l+s-1) !\left(\frac{a b}{k}\right)^{s} C^{(m-s)}\left(\frac{k \bar{b}}{a}\right) \\
& -\frac{l}{a^{m-l}} \sum_{s=0}^{m-2}\left(\begin{array}{c}
m \\
s
\end{array}\right) \frac{(a b)^{s}(l+s-1) ! B_{m-s}}{(2 \pi i k)^{l+s}}-\frac{l(l+m-1) ! a^{l} b^{m}}{(2 \pi i k)^{l+m}} .
\end{aligned}
$$

Case $3: a \nmid k$ but $b \mid k$. Since the formula for $c_{k}(a, b)$ is symmetric in $a$ and $b$, we have

(24)

$$
\begin{aligned}
= & \frac{a^{l-m} l m}{k^{l}(2 \pi i)^{l+m}} \sum_{s=0}^{m-1}\left(\begin{array}{c}
m-1 \\
s
\end{array}\right)(-1)^{m+s-1}(l+s-1) !\left(\frac{a b}{k}\right)^{s} C^{(m-s)}\left(\frac{k \bar{b}}{a}\right) \\
& -\frac{m}{b^{l-m}} \sum_{s=0}^{l-2}\left(\begin{array}{l}
l \\
s
\end{array}\right) \frac{(a b)^{s}(m+s-1) ! B_{l-s}}{(2 \pi i k)^{m+s}}-\frac{m(l+m-1) ! a^{l} b^{m}}{(2 \pi i k)^{l+m}}
\end{aligned}
$$

for this case.

Case $4: a \mid k$ and $b \mid k$. In this case $f(z)$ has poles of order $l+1$ at $z=-k \bar{a} / b$ and $m+1$ at $z=k \bar{b} / a$, so that

$$
\begin{aligned}
c_{k}(a, b) & -\frac{l}{a^{m-l}} \sum_{s=0}^{m-2}\left(\begin{array}{c}
m \\
s
\end{array}\right) \frac{(a b)^{s}(l+s-1) ! B_{m-s}}{(2 \pi i k)^{l+s}}-\frac{l(l+m-1) ! a^{l} b^{m}}{(2 \pi i k)^{l+m}} \\
& -\frac{m}{b^{l-m}} \sum_{s=0}^{l-2}\left(\begin{array}{l}
l \\
s
\end{array}\right) \frac{(a b)^{s}(m+s-1) ! B_{l-s}}{(2 \pi i k)^{m+s}}-\frac{m(l+m-1) ! a^{l} b^{m}}{(2 \pi i k)^{l+m}} .
\end{aligned}
$$

Thus, after a slight rearrangement, (20), (22), (23), (24) and (25) give

$$
\begin{aligned}
\bar{B}_{l}(a x) \bar{B}_{m}(b x) \sim \frac{(-1)^{m-1} l ! m ! B_{l+m}}{a^{m} b^{l}(l+m) !} & \\
-\sum_{\substack{k=-\infty \\
a \mid k}}^{\infty}\left\{\frac{l}{a^{m-l}} \sum_{s=0}^{m-2}\left(\begin{array}{c}
m \\
s
\end{array}\right) \frac{(a b)^{s}(l+s-1) ! B_{m-s}}{(2 \pi i k)^{l+s}}\right. & \left.+\frac{l(l+m-1) ! a^{l} b^{m}}{(2 \pi i k)^{l+m}}\right\} e(k x)
\end{aligned}
$$




$$
\begin{aligned}
& -\sum_{\substack{k=-\infty \\
b \mid k}}^{\infty}\left\{\frac{m}{b^{l-m}} \sum_{s=0}^{l-2}\left(\begin{array}{l}
l \\
s
\end{array}\right) \frac{(a b)^{s}(m+s-1) ! B_{l-s}}{(2 \pi i k)^{m+s}}\right. \\
& \left.\quad+\frac{m(l+m-1) ! a^{l} b^{m}}{(2 \pi i k)^{l+m}}\right\} e(k x) \\
& -\sum_{\substack{k=-\infty \\
a \nmid k}}^{\infty} \frac{a^{l-m} l m}{k^{l}(2 \pi i)^{l+m}} \\
& \quad \times \sum_{s=0}^{m-1}\left(\begin{array}{c}
m-1 \\
s
\end{array}\right)(-1)^{m+s}(l+s-1) !\left(\frac{a b}{k}\right)^{s} C^{(m-s)}\left(\frac{k \bar{b}}{a}\right) e(k x) \\
& -\sum_{\substack{k=-\infty \\
b \nmid k}}^{\infty} \frac{b^{m-l} l m}{k^{m}(2 \pi i)^{l+m}} \\
& \quad \times \sum_{s=0}^{l-1}\left(\begin{array}{c}
l-1 \\
s
\end{array}\right)(-1)^{l+s}(m+s-1) !\left(\frac{a b}{k}\right)^{s} C^{(l-s)}\left(\frac{k \bar{a}}{b}\right) e(k x) .
\end{aligned}
$$

\section{The triple Franel-Kluyver integral. Let}

$$
I_{l, m, n}(a, b, c)=\int_{0}^{1} \bar{B}_{l}(a x) \bar{B}_{m}(b x) \bar{B}_{n}(c x) d x
$$

where we may assume $a, b$ and $c$ are pairwise coprime and that $l+m+n$ is even since the integral is zero otherwise. Then we have

THEOREM 1.

(27) $\quad I_{l, m, n}(a, b, c)$

$=\frac{(-1)^{l+n} l ! m ! n ! a^{l-1}}{c^{l}} \sum_{s=0}^{m}\left(\begin{array}{c}l+s-1 \\ s\end{array}\right)\left(\frac{b}{c}\right)^{s}(-1)^{s+1} \frac{S_{m-s, l+n+s}(c \bar{b}, a)}{(m-s) !(l+n+s) !}$

$+\frac{(-1)^{m+n} l ! m ! n ! b^{m-1}}{c^{m}} \sum_{s=0}^{l}\left(\begin{array}{c}m+s-1 \\ s\end{array}\right)\left(\frac{a}{c}\right)^{s}(-1)^{s+1} \frac{S_{l-s, m+n+s}(c \bar{a}, b)}{(l-s) !(m+n+s) !}$.

Pr o of. We apply Parseval's formula to the functions $\bar{B}_{l}(a x) \bar{B}_{m}(b x)$ and $\bar{B}_{n}(c x)$ to obtain

(28) $\quad I_{l, m, n}(a, b, c)$

$$
=\sum_{a \mid k, k=h c}^{\prime}\left\{\frac{l n !}{a^{m-l}} \sum_{s=0}^{m-2}\left(\begin{array}{c}
m \\
s
\end{array}\right) \frac{(a b)^{s}(l+s-1) ! B_{m-s}}{(2 \pi i)^{l+n+s} h^{n} k^{l+s}}+\frac{l(l+m-1) ! n ! a^{l} b^{m}}{(2 \pi i)^{l+m+n} h^{n} k^{l+m}}\right\}
$$




$$
\begin{gathered}
+\sum_{b \mid k, k=h c}^{\prime}\left\{\frac{m n !}{b^{l-m}} \sum_{s=0}^{l-2}\left(\begin{array}{l}
l \\
s
\end{array}\right) \frac{(a b)^{s}(m+s-1) ! B_{l-s}}{(2 \pi i)^{m+n+s} h^{n} k^{m+s}}+\frac{m(l+m-1) ! n ! a^{l} b^{m}}{(2 \pi i)^{l+m+n} h^{n} k^{l+m}}\right\} \\
+\sum_{a \nmid k, k=h c}^{\prime}\left\{\frac{a^{l-m} l m n !}{(2 \pi i)^{l+m+n}}\right. \\
\left.\quad \times \sum_{s=0}^{m-1}\left(\begin{array}{c}
m-1 \\
s
\end{array}\right) \frac{(-1)^{m+s}(l+s-1) !(a b)^{s}}{h^{n} k^{l+s}} C^{(m-s)}\left(\frac{k \bar{b}}{a}\right)\right\} \\
+\sum_{b \nmid k, k=h c}^{\prime}\left\{\frac{b^{m-l} l m n !}{(2 \pi i)^{l+m+n}}\right. \\
\left.\times \sum_{s=0}^{l-1}\left(\begin{array}{c}
l-1 \\
s
\end{array}\right) \frac{(-1)^{l+s}(m+s-1) !(a b)^{s}}{h^{n} k^{m+s}} C^{(l-s)}\left(\frac{k \bar{a}}{b}\right)\right\} .
\end{gathered}
$$

In the first sum here, since $a \mid k$ and $(a, c)=1$, we must have $a \mid h$ so we put $h=d a$; then $k=c d a$ and the first sum is

$$
\begin{aligned}
\frac{l n !}{a^{m+n} c^{l}} \sum_{s=0}^{m-2}\left(\begin{array}{c}
m \\
s
\end{array}\right)\left(\frac{b}{c}\right)^{s} \frac{(l+s-1) ! B_{m-s}}{(2 \pi i)^{l+n+s}} \sum_{d=-\infty}^{\infty} \frac{1}{d^{l+n+s}} & \\
& \quad+\frac{l(l+m-1) ! n ! b^{m}}{(2 \pi i)^{l+m+n} a^{m+n} c^{l+m}} \sum_{d=-\infty}^{\infty} \frac{1}{d^{l+m+n}} .
\end{aligned}
$$

Using (19) we can write this as

$$
\begin{aligned}
-\frac{l n !}{a^{m+n} c^{l}} \sum_{s=0}^{m}\left(\begin{array}{c}
m \\
s
\end{array}\right)\left(\frac{b}{c}\right)^{s} \frac{(l+s-1) ! B_{m-s} B_{n+l+s}}{(n+l+s) !} \\
=-\frac{l ! m ! n !}{a^{m+n} c^{l}} \sum_{s=0}^{m}\left(\begin{array}{c}
l+s-1 \\
s
\end{array}\right)\left(\begin{array}{l}
b \\
c
\end{array}\right)^{s} \frac{B_{m-s} B_{n+l+s}}{(m-s) !(n+l+s) !} .
\end{aligned}
$$

Similarly, the second sum in (28) gives

$$
-\frac{l ! m ! n !}{b^{l+n} c^{m}} \sum_{s=0}^{l}\left(\begin{array}{c}
m+s-1 \\
s
\end{array}\right)\left(\frac{a}{c}\right)^{s} \frac{B_{l-s} B_{n+m+s}}{(l-s) !(n+m+s) !} .
$$

For the third sum in (28) we have $a \nmid k$ and $k=h c$ so we put $h=d a+r$, $0<r<a$; then this sum is

$$
\begin{aligned}
\frac{a^{l-m} l m n !}{(2 \pi i)^{l+m+n} c^{l}} \sum_{s=0}^{m-1}\left(\frac{a b}{c}\right)^{s}\left(\begin{array}{c}
m-1 \\
s
\end{array}\right)(-1)^{m+s}(l+s-1) ! \\
\quad \times \sum_{r=1}^{a-1} C^{(m-s)}\left(\frac{r c \bar{b}}{a}\right) \sum_{d=-\infty}^{\infty} \frac{1}{(d a+r)^{l+n+s}} .
\end{aligned}
$$


Now, from (9),

$$
\sum_{d=-\infty}^{\infty} \frac{1}{(d a+r)^{l+n+s}}=\frac{(-1)^{l+n+s-1}}{a^{l+n+s}(l+n+s-1) !} C^{(l+n+s)}\left(\frac{r}{a}\right)
$$

so the third sum is

$$
\begin{aligned}
& -\frac{l m n !}{(2 \pi i)^{l+m+n} a^{m+n} c^{l}} \\
& \quad \times \sum_{s=0}^{m-1}\left(\begin{array}{c}
m-1 \\
s
\end{array}\right)\left(\frac{b}{c}\right)^{s} \frac{(l+s-1) !}{(l+n+s-1) !} \sum_{r=0}^{a-1} C^{(m-s)}\left(\frac{r c \bar{b}}{a}\right) C^{(l+n+s)}\left(\frac{r}{a}\right) \\
& +\frac{l m n !}{(2 \pi i)^{l+m+n} a^{m+n} c^{l}} \\
& \quad \times \sum_{s=0}^{m-1}\left(\begin{array}{c}
m-1 \\
s
\end{array}\right) \frac{(l+s-1) !}{(l+n+s-1) !}\left(\frac{b}{c}\right)^{s} C^{(m-s)}(0) C^{(l+n+s)}(0),
\end{aligned}
$$

which, from (8) and (10), is

(31)

$$
\begin{aligned}
& \frac{(-1)^{l+n} l ! m ! n ! a^{l-1}}{c^{l}} \\
& \quad \times \sum_{s=0}^{m-1}\left(\begin{array}{c}
l+s-1 \\
s
\end{array}\right)\left(\frac{b}{c}\right)^{s}(-1)^{s+1} \frac{S_{m-s, l+n+s}(\bar{b}, a)}{(m-s) !(l+n+s) !} \\
& \quad+\frac{l ! m ! n !}{a^{m+n} c^{l}} \sum_{s=0}^{m-1}\left(\begin{array}{c}
l+s-1 \\
s
\end{array}\right)\left(\frac{b}{c}\right)^{s} \frac{B_{m-s} B_{l+n+s}}{(m-s) !(l+n+s) !} .
\end{aligned}
$$

Similarly the fourth sum in (28) is

$$
\begin{aligned}
& \frac{(-1)^{m+n} l ! m ! n ! b^{m-1}}{c^{m}} \\
& \quad \times \sum_{s=0}^{l-1}\left(\begin{array}{c}
m+s-1 \\
s
\end{array}\right)\left(\frac{a}{c}\right)^{s}(-1)^{s+1} \frac{S_{l-s, m+n+s}(c \bar{a}, b)}{(l-s) !(m+n+s) !} \\
& +\frac{l ! m ! n !}{b^{l+n} c^{m}} \sum_{s=0}^{l-1}\left(\begin{array}{c}
m+s-1 \\
s
\end{array}\right)\left(\frac{a}{c}\right)^{s} \frac{B_{l-s} B_{m+n+s}}{(l-s) !(m+n+s) !} .
\end{aligned}
$$

Thus, (29)-(32) give

$$
\begin{aligned}
& I_{l, m, n}(a, b, c) \\
& =\frac{(-1)^{l+n} l ! m ! n ! a^{l-1}}{c^{l}} \sum_{s=0}^{m-1}\left(\begin{array}{c}
l+s-1 \\
s
\end{array}\right)\left(\frac{b}{c}\right)^{s}(-1)^{s+1} \frac{S_{m-s, l+n+s}(c \bar{b}, a)}{(m-s) !(l+n+s) !}
\end{aligned}
$$




$$
\begin{aligned}
& +\frac{(-1)^{m+n} l ! m ! n ! b^{m-1}}{c^{m}} \sum_{s=0}^{l-1}\left(\begin{array}{c}
m+s-1 \\
s
\end{array}\right)\left(\frac{a}{c}\right)^{s}(-1)^{s+1} \frac{S_{l-s, m+n+s}(c \bar{a}, b)}{(l-s) !(m+n+s) !} \\
& -\frac{l ! m ! n !}{(l+m+n) !} \frac{b^{m}}{a^{m+n} c^{l+m}}\left(\begin{array}{c}
l+m-1 \\
m
\end{array}\right) B_{l+m+n} \\
& -\frac{l ! m ! n !}{(l+m+n) !} \frac{a^{l}}{b^{l+n} c^{l+m}}\left(\begin{array}{c}
l+m-1 \\
l
\end{array}\right) B_{l+m+n},
\end{aligned}
$$

which is equivalent to $(27)$.

\section{Shifted triple integrals. Let}

$$
I(a, b, c ; \theta)=\int_{0}^{1} \bar{B}_{1}(a x) \bar{B}_{1}(b x) \bar{B}_{1}(c x+\theta) d x
$$

where we may assume $a, b$ and $c$ are pairwise coprime and, since the integrand has period 1 in $\theta$, that $0<\theta<1$. In order to evaluate this integral we shall require the following lemma.

Lemma 2. For $\alpha \notin \mathbb{Z}$ and all real $x$ we have

$$
\begin{aligned}
& \sum_{n=-\infty}^{\infty} \frac{e((n+\alpha) x)}{(n+\alpha)^{k}}=\delta_{k}(x)+\frac{e([x] \alpha) 2^{k-1}(i \pi)^{k}\{x\}^{k-1}}{(k-1) !} \\
& +\frac{e([x] \alpha)}{(k-1) !} \sum_{s=0}^{k-1}\left(\begin{array}{c}
k-1 \\
s
\end{array}\right)(-1)^{s} C^{(s+1)}(\alpha)(2 \pi i\{x\})^{k-1-s}
\end{aligned}
$$

where $\{x\}$ denotes the fractional part of $x$ and

$$
\delta_{k}(x)= \begin{cases}1 & \text { if } k=1 \text { and } x \in \mathbb{Z}, \\ 0 & \text { else. }\end{cases}
$$

Proof. We begin with the Fourier series

$$
e(\alpha(1 / 2-\{x\})) \sim \frac{\sin \pi \alpha}{\pi \alpha}+\frac{2 \sin \pi \alpha}{\pi} \sum_{n=1}^{\infty} \frac{\alpha \cos 2 \pi n x-i n \sin 2 \pi n x}{\alpha^{2}-n^{2}} .
$$

The function on the left has bounded variation on any interval $[a, b]$ and is continuous for all $x \in \mathbb{R} \backslash \mathbb{Z}$. It follows that we may replace $\sim$ by $=$ above provided we add the term $\delta_{1}(x) i \sin \pi \alpha$ on the right to take care of integral $x$. We write, formally,

$$
e(\alpha(1 / 2-\{x\}))=\delta_{1}(x) i \sin \pi \alpha+\sum_{n=-\infty}^{\infty} \frac{\sin \pi \alpha}{\pi(n+\alpha)} e(n x)
$$

with the understanding that the terms involving $\pm n$ are bracketed together. We multiply through by $\pi e(\alpha x) / \sin \pi \alpha$ to obtain (33) in the case $k=1$. 
We may write the summand on the right-hand side of (34) in the form

$$
\frac{(\alpha-n) e(n x)+(\alpha+n) e(-n x)}{2\left(\alpha^{2}-n^{2}\right)} .
$$

Let $x \in[0,1)$. We multiply (34) by $\pi e(\alpha x) / \sin \pi \alpha$ and integrate term-byterm from 0 to $y$. This step is justified by $\S 13.53$ of Titchmarsh [7], and we obtain, for $y \in[0,1)$,

$$
\begin{aligned}
\frac{\pi e(\alpha / 2)}{\sin \pi \alpha} y & =\frac{e(\alpha y)-1}{2 \pi i \alpha^{2}}+\sum_{n=1}^{\infty}\left(\frac{e((\alpha+n) y)-1}{2 \pi i(\alpha+n)^{2}}+\frac{e((\alpha-n) y)-1}{2 \pi i(\alpha-n)^{2}}\right) \\
& =\frac{1}{2 \pi i}\left(\sum_{n=-\infty}^{\infty} \frac{e((\alpha+n) y)}{(\alpha+n)^{2}}-\pi^{2} \operatorname{cosec}^{2} \pi \alpha\right) .
\end{aligned}
$$

We extend this periodically to obtain (33) in the case $k=2$. We now proceed by induction on $k$, and from this point the term-by-term integrations may be justified by uniform convergence. Accordingly, assume that $k \geq 2, x \in[0,1)$ and (33) holds. Integrating from 0 to $y$ gives

$$
\begin{aligned}
& \sum_{n=-\infty}^{\infty} \frac{e((n+\alpha) y)-1}{(n+\alpha)^{k+1}}=2 \pi i\left\{\frac{2^{k-1}(i \pi)^{k} y^{k}}{k !}\right. \\
&\left.+\frac{1}{(k-1) !} \sum_{s=0}^{k-1}\left(\begin{array}{c}
k-1 \\
s
\end{array}\right) \frac{(-1)^{s} C^{(s+1)}(\alpha)(2 \pi i)^{k-1-s} y^{k-s}}{k-s}\right\}
\end{aligned}
$$

so that

$$
\begin{aligned}
\sum_{n=-\infty}^{\infty} \frac{e((n+\alpha) y)}{(n+\alpha)^{k+1}}= & \frac{2^{k}(i \pi)^{k+1} y^{k}}{k !}+\sum_{n=-\infty}^{\infty} \frac{1}{(n+\alpha)^{k+1}} \\
& +\frac{1}{k !} \sum_{s=0}^{k-1}\left(\begin{array}{l}
k \\
s
\end{array}\right)(-1)^{s} C^{(s+1)}(\alpha)(2 \pi i y)^{k-s}
\end{aligned}
$$

Therefore, since

$$
C^{(k+1)}(\alpha)=(-1)^{k} k ! \sum_{n=-\infty}^{\infty} \frac{1}{(n+\alpha)^{k+1}}
$$

equation (33) holds for $k+1$ and hence for all $k \geq 1$.

We see from (9) that (33) also holds when $x=0$ and therefore, since

$$
\sum_{n=-\infty}^{\infty} \frac{e((n+\alpha) x)}{(n+\alpha)^{k}}=\sum_{n=-\infty}^{\infty} \frac{e((n+\alpha)\{x\}) e([x] \alpha)}{(n+\alpha)^{k}}
$$

(33) follows for $x \in \mathbb{R}$. 
TheOREM 2. For $a, b, c$ pairwise coprime integers and $0<\theta<1$,

$$
\begin{aligned}
I(a, b, c ; \theta)= & \frac{\{a \theta\}}{a c} S_{1,1}(\bar{b} c, a ;[a \theta] / a)+\frac{\{b \theta\}}{b c} S_{1,1}(\bar{a} c, b ;[b \theta] / b) \\
& +\frac{1}{2 c}\left\{S_{1,2}(\bar{b} c, a ;[a \theta] / a)+S_{1,2}(\bar{a} c, b ;[b \theta] / b)\right\} \\
& +\frac{\{a \theta\}}{2 a c} \bar{B}_{1}\left(\frac{[a \theta] b \bar{c}}{a}\right)+\frac{\{b \theta\}}{2 b c} \bar{B}_{1}\left(\frac{[b \theta] a \bar{c}}{b}\right) \\
& -\frac{1}{6 a^{2} b^{2} c^{2}}\left\{b^{3} \bar{B}_{3}(a \theta)+a^{3} \bar{B}_{3}(b \theta)\right\} .
\end{aligned}
$$

Pr o of. Since

$$
\bar{B}_{1}(c x+\theta) \sim-\sum_{g=-\infty}^{\infty} \frac{e(g \theta) e(g c x)}{2 \pi i g}
$$

and, from (26),

$$
\begin{aligned}
\bar{B}_{1}(a x) \bar{B}_{1}(b x) \sim & \frac{1}{12 a b}+\sum_{\substack{h=-\infty \\
a \mid h}}^{\infty} \frac{a b}{4 \pi^{2} h^{2}} e(-h x)+\sum_{\substack{h=-\infty \\
b \mid h}}^{\infty} \frac{a b}{4 \pi^{2} h^{2}} e(-h x) \\
& -\sum_{\substack{h=-\infty \\
a \nmid h}}^{\infty} \frac{1}{4 \pi h} \cot \left(\frac{\pi h \bar{b}}{a}\right) e(-h x) \\
& -\sum_{\substack{h=-\infty \\
b \nmid h}}^{\infty} \frac{1}{4 \pi h} \cot \left(\frac{\pi h \bar{a}}{b}\right) e(-h x),
\end{aligned}
$$

Parseval's formula gives

$$
\begin{aligned}
I(a, b, c ; \theta)= & \sum^{\prime}\left\{-\frac{e(g \theta) a b}{8 \pi^{3} i g h^{2}} ; a \mid h, h=g c\right\} \\
& +\sum^{\prime}\left\{-\frac{e(g \theta) a b}{8 \pi^{3} i g h^{2}} ; b \mid h, h=g c\right\} \\
& +\sum^{\prime}\left\{-\frac{e(g \theta)}{8 \pi^{2} i g h} \cot \left(\frac{\bar{b} h \pi}{a}\right) ; a \nmid h, h=g c\right\} \\
& +\sum^{\prime}\left\{-\frac{e(g \theta)}{8 \pi^{2} i g h} \cot \left(\frac{\bar{a} h \pi}{b}\right) ; b \nmid h, h=g c\right\} .
\end{aligned}
$$

For the first sum we let $g=l a$, then $h=c l a$ and the sum is

$$
-\sum_{l=-\infty}^{\infty} \frac{e(l a \theta) b}{8 \pi^{3} i a^{2} c^{2} l^{3}}=-\frac{b}{8 \pi^{3} i a^{2} c^{2}} \sum_{l=-\infty}^{\infty} \frac{e(l a \theta)}{l^{3}}=-\frac{b}{6 a^{2} c^{2}} \bar{B}_{3}(a \theta) .
$$


Similarly, the second sum is

$$
-\frac{a}{b^{2} c^{2}} \bar{B}_{3}(b \theta) \text {. }
$$

In the third sum in (36) we must have $a \nmid g$. Put $g=l a+r, 0<r<a$; then the sum is

$$
\frac{1}{8 \pi^{2} i c} \sum_{r=1}^{a-1} \cot \left(\frac{c r \bar{b} \pi}{a}\right) \sum_{l=-\infty}^{\infty} \frac{e(l a \theta+r \theta)}{(l a+r)^{2}} .
$$

Now, by Lemma 2,

$$
\begin{aligned}
& \sum_{l=-\infty}^{\infty} \frac{e(l a \theta+r \theta)}{(l a+r)^{2}} \\
& \quad=\frac{e([a \theta] r / a)}{a^{2}}\left\{2(i \pi)^{2}\{a \theta\}+2 \pi i\{a \theta\} C^{(1)}\left(\frac{r}{a}\right)-C^{(2)}\left(\frac{r}{a}\right)\right\},
\end{aligned}
$$

so that (39) is

$$
\begin{aligned}
\frac{i\{a \theta\}}{4 \pi c a^{2}} \sum_{r=1}^{a-1} C^{(1)}\left(\frac{c r \bar{b}}{a}\right) e\left(\frac{[a \theta] r}{a}\right) \\
+\frac{\{a \theta\}}{4 \pi^{2} c a^{2}} \sum_{r=1}^{a-1} C^{(1)}\left(\frac{c r \bar{b}}{a}\right) C^{(1)}\left(\frac{r}{a}\right) e\left(\frac{[a \theta] r}{a}\right) \\
+\frac{i}{8 \pi^{3} c a^{2}} \sum_{r=1}^{a-1} C^{(1)}\left(\frac{c r \bar{b}}{a}\right) C^{(2)}\left(\frac{r}{a}\right) e\left(\frac{[a \theta] r}{a}\right) .
\end{aligned}
$$

From (11) we have

$$
\bar{B}_{n}\left(\frac{h \mu}{k}+x\right)=\frac{n}{k^{n}}\left(\frac{i}{2 \pi}\right)^{n} \sum_{\lambda=0}^{k-1} C^{(n)}\left(\frac{\lambda}{k}\right) e\left(\frac{\lambda(h \mu+k x)}{k}\right)
$$

so that we can also write

$$
S_{m, n}(h, k ; x)=\frac{(-1)^{(n-m) / 2} m n}{(2 \pi)^{m+n} k^{m+n-1}} \sum_{\lambda=0}^{k-1} e(\lambda x) C^{(m)}\left(\frac{h \lambda}{k}\right) C^{(n)}\left(\frac{\lambda}{k}\right)
$$

and the third sum in (36) is

(41) $\frac{\{a \theta\}}{2 a c} \bar{B}_{1}\left(\frac{[a \theta] b \bar{c}}{a}\right)+\frac{\{a \theta\}}{a c} S_{1,1}(\bar{b} c, a ;[a \theta] / a)+\frac{1}{2 c} S_{1,2}(\bar{b} c, a ;[a \theta] / a)$.

Similarly, the fourth sum is

$$
\frac{\{b \theta\}}{2 b c} \bar{B}_{1}\left(\frac{[b \theta] a \bar{c}}{b}\right)+\frac{\{b \theta\}}{b c} S_{1,1}(\bar{a} c, b ;[b \theta] / b)+\frac{1}{2 c} S_{1,2}(\bar{a} c, b ;[b \theta] / b),
$$

so that, from (37), (38), (41) and (42) we obtain (35) as required. 
Notice that the integral on the left-hand side of (35) is bounded in absolute value by $1 / 32$ for any $a, b$ and $c$ (using Hölder's inequality), whereas there are terms on the right-hand side which can be very large in certain cases. For example, if $a$ is very large in comparison with $b$ and $c$ then there must be cancellation between the terms

$$
\frac{1}{2 c} S_{1,2}(\bar{b} c, a ;[a \theta] / a)-\frac{a}{6 b^{2} c^{2}} \bar{B}_{3}(b \theta)
$$

which suggests the existence of reciprocity relations for the homogeneous Dedekind-Rademacher sums

$$
S_{m, n}(c, b, a ; x)=S_{m, n}(\bar{b} c, a ; x) .
$$

In fact such relations do exist, but the appropriate choice in a particular problem depends on the relative magnitudes of $a, b$ and $c$ : we do not have a single formula for $I(a, b, c ; \theta)$ in terms of Dedekind sums in which all the terms are uniformly bounded. I hope to consider this matter further in a later paper.

Acknowledgements. The author is indebted to the UK Science and Engineering Research Council for the award of a Research Studentship.

\section{References}

[1] L. Carlitz, Some theorems on generalized Dedekind-Rademacher sums, Pacific J. Math. 75 (1978), 347-358.

[2] J. Franel, Les suites de Farey et le problème des nombres premiers, Göttinger Nachr. 1924, 198-201.

[3] G. R. H. Greaves, R. R. Hall, M. N. Huxley and J. C. Wilson, Multiple Franel integrals, Mathematika 40 (1993), 50-69.

[4] R. R. Hall, The distribution of squarefree numbers, J. Reine Angew. Math. 394 (1989), 107-117.

[5] -, Large irregularities in sets of multiples and sieves, Mathematika 37 (1990), 119-135.

[6] J. C. Kluyver, An analytical expression for the greatest common divisor of two integers, Proc. Roy. Acad. Amsterdam, Vol. V.II, (1903), 658-662.

[7] E. C. Titchmarsh, The Theory of Functions, University Press, Oxford, 1939.

[8] E. T. Whittaker and G. N. Watson, Modern Analysis, University Press, Cambridge, 1945.

DEPARTMENT OF MATHEMATICS

UNIVERSITY OF YORK

YORK YO1 5DD, U.K. 